\title{
ATIVIDADE ANTIMICROBIANA DE MÉIS PRODUZIDOS POR MELIPONÍNEOS NATIVOS DO PARANÁ (BRASIL)
}

\author{
DÉBORA MARIA BORSATO* \\ LUÍS ANTÔNIO ESMERINO** \\ PAULO VITOR FARAGO*** \\ MARILIS DALLARMI MIGUEL**** \\ OBDULIO GOMES MIGUEL ${ }^{* * * *}$
}

\begin{abstract}
O objetivo deste trabalho foi estudar a atividade antibacteriana e antifúngica de méis in natura, elaborados por abelhas nativas sem ferrão e de extratos fenólicos (EFM) obtidos a partir dessas amostras. A atividade antimicrobiana de 21 amostras de mel, coletadas no Estado do Paraná (Brasil), foi avaliada pelo método de microdiluição em caldo, determinando-se a concentração inibitória mínima (MIC) contra as cepas de Escherichia coli ATCC 10530, Staphylococcus aureus ATCC 25923 e Candida albicans ATCC 10231. As amostras de mel apresentaram maior atividade antimicrobiana para as bactérias $E$. coli $\left(\mathrm{MIC}_{90} \geq 3,12 \%\right)$ e $S$. aureus $\left(\mathrm{MIC}_{90} \geq 1,56 \%\right)$ em comparação aos valores obtidos para a levedura C. albicans $\left(\mathrm{MIC}_{90} \geq 12,5 \%\right)$. Os EFM revelaram menor atividade antimicrobiana em relação aos méis in natura, pois apenas nove amostras de EFM inibiram o crescimento de $S$. aureus em $10 \mathrm{mg} \cdot \mathrm{mL}^{-1}$. Tais resultados permitem inferir que o mel de abelhas sem ferrão constitui alimento com propriedades funcionais e que apresenta potencial terapêutico contra infecções.
\end{abstract}

* Doutoranda em Ciências Farmacêuticas, Programa de Pós-graduação em Ciências Farmacêuticas, Universidade Federal do Paraná (UFPR), Curitiba, PR (e-mail: dmborsato@yahoo.com.br).

** Doutor em Odontologia, Professor Associado, Departamento de Análises Clínicas e Toxicológicas, Universidade Estadual de Ponta Grossa (UEPG), Ponta Grossa, PR (e-mail: esmerino@uepg.br).

*** Doutor em Química, Professor Adjunto, Departamento de Ciências Farmacêuticas, UEPG, Ponta Grossa, PR (e-mail: pvfarago@uepg.br).

**** Doutora em Agronomia, Professora Associada, Departamento de Farmácia, UFPR, Curitiba, PR (e-mail: dallarmi@ufpr.br).

***** Doutor em Química, Professor Adjunto, Departamento de Farmácia, UFPR, Curitiba, PR (e-mail: obdulio@ ufpr.br). 


\section{INTRODUÇÃo}

O mel, alimento natural e rico em nutrientes essenciais, é produzido por diferentes espécies de abelhas (CRANE, 1997). Além de alimento energético e adoçante natural, o mel apresenta atividades biológicas, como antimicrobiana, antifúngica, antioxidante, antiviral, antiparasitária, antiinflamatória, anticâncer e imunossupressora (IRISH et al., 2006; BOGDANOV et al., 2008; ALVAREZSUAREZ et al., 2010). A utilização do mel contra infecções bacterianas faz parte da medicina tradicional há séculos. No entanto, foi substituída por antibióticos sintéticos e/ou semissintéticos (MOLAN, SMITH e REID, 1988).

Devido ao uso indiscriminado e irracional de antibióticos há mais de 50 anos, as bactérias têm progressivamente desenvolvido resistência a esses medicamentos (HSUEH, CHEN e LUH, 2005). Os relatos crescentes de resistência bacteriana a antibióticos e a incidência de efeitos colaterais ocasionados por certos produtos farmacêuticos têm conduzido à investigação de novos compostos com ação antimicrobiana a partir de diferentes produtos naturais, como o mel, em substituição à terapia antibiótica convencional (GAREDEW, SCHMOLZ e LAMPRECHT, 2004).

O mel produzido pela abelha Apis mellifera L., o mais comum, é amplamente estudado (MARCHINI, MORETI e SILVEIRA NETO, 2003; BORSATO et al., 2010) e usado pela população. Particularmente na região Neotropical, além das abelhas africanizadas, várias espécies de abelhas indígenas sem ferrão ou meliponíneos (como Melipona spp., Scaptotrigona spp. e Trigona spp.) elaboram mel diferenciado. As características sensoriais e físico-químicas particulares do mel de abelhas sem ferrão tornam seu valor comercial mais elevado, quando comparado ao mel tradicional (GAREDEW, SCHMOLZ e LAMPRECHT, 2004; SOUZA et al., 2004a; TORRES et al., 2004; ALMEIDA-MURADIAN, MATSUDA e BASTOS, 2007; GUERRINI et al., 2009).

O mel das abelhas sem ferrão tem sido utilizado popularmente como alimento com finalidades medicinais, principalmente em zonas rurais e entre indígenas, que the atribuem propriedades terapêuticas específicas, como o tratamento de infecções (POSEY, 1987; CORTOPASSI-LAURINO e GELLI, 1991). Nesse sentido, alguns autores avaliaram o efeito antimicrobiano de méis produzidos por espécies de abelhas indígenas sem ferrão, provenientes de diferentes países. Torres et al. (2004) evidenciaram a ação do mel de Tetragonisca angustula, proveniente da Colômbia, frente a bactérias Gram-positivas (Bacillus brevis, Bacillus megaterium, Bacillus subtilis e Micrococcus luteus) e Gram-negativas (Escherichia coli e Pseudomonas syringae). Já os fungos Aspergillus niger, Penicillium chrysogenum e Trichoderma viride não foram sensíveis ao tratamento com o mel da referida espécie. Garedew, Schmolz e Lamprecht (2004) ao investigarem a ação antibacteriana do mel de Trigona spp., proveniente da Etiópia, constataram que as duas amostras de mel testadas foram eficientes contra as mesmas cepas de bactérias Gram-positivas e Gram-negativas. Dardón e Enríquez (2008) comprovaram o efeito antimicrobiano de amostras de mel de nove espécies de meliponíneos da Guatemala para diversas bactérias e leveduras. Irish et al. (2008) confirmaram o efeito do mel da abelha australiana Trigona carbonaria frente ao micro-organismo Staphylococcus aureus. Guerrini et al. (2009) demonstraram o potencial antibacteriano do mel de abelhas nativas do Equador para Enterococcus faecalis, E. coli, Pseudomonas aeruginosa e S. aureus subsp. aureus. Boorn et al. (2010) comprovaram que o mel de T. carbonaria apresentou amplo espectro de atividade antibacteriana. Entretanto, a atividade contra Candida mostrou-se limitada.

No Brasil, a atividade antimicrobiana de méis produzidos por algumas espécies de abelhas indígenas sem ferrão foi também investigada. Cortopassi-Laurino e Gelli (1991) constataram que do total de ensaios de antibiose realizados com mel de meliponíneos, $50 \%$ dos micro-organismos mostraram-se sensíveis. Miorin et al. (2003) comprovaram a atividade antibacteriana de amostras de mel e própolis produzidos por $T$. angustula diante de S. aureus. Gonçalves, Alves Filho e Menezes (2005) avaliaram a atividade antimicrobiana do mel da abelha Nannotrigona testaceicornis, sendo que os micro-organismos E. coli, Proteus spp., P. aeruginosa, Streptococcus pyogenes, bem como Staphylococcus spp. coagulase negativa, revelaram-se sensíveis frente à ação do mel 
estudado. Entretanto, Enterobacter aerogenes, Enterobacter cloacae, Proteus mirabilis, S. aureus e Streptococcus a-hemolítico mostraram-se resistentes à ação do produto. Alves et al. (2008) comprovaram que o uso tópico de mel de Melipona subnitida em feridas infectadas da pele de ratos estimulou a resposta imunológica, reduziu significativamente a infecção por bactérias Gramnegativas e Gram-positivas, bem como o tempo de cicatrização. Considerando que existem mais de 192 espécies de meliponíneos no Brasil (BALLIVIÁN, 2008) pode-se estabelecer que os estudos já realizados, embora promissores, não contemplam a diversidade dos méis produzidos por essas espécies.

Com base na literatura consultada não foram verificados trabalhos relativos à atividade antimicrobiana de méis de abelhas indígenas sem ferrão elaborados pelas espécies Melipona bicolor, Melipona marginata, Melipona quadrifasciata, Melipona rufiventris, Scaptotrigona bipunctata, Scaptotrigona xantotricha e Tetragona clavipes, além de méis de Tetragonisca angustula provenientes do Estado do Paraná. Assim, com o propósito de ampliar o conhecimento sobre o potencial medicinal de méis obtidos a partir de diferentes meliponíneos nativos do Brasil, o objetivo desse trabalho foi avaliar a atividade antibacteriana e antifúngica desses produtos in natura, provenientes do Estado do Paraná, e de extratos metanólicos obtidos a partir das amostras.

\section{MATERIAL E MÉTODOS}

\subsection{AMOSTRAS DE MEL}

O total de 21 amostras de mel de meliponíneos foi coletado em diferentes municípios do Estado do Paraná (Brasil), entre os anos de 2010 e 2011, conforme detalhado na Tabela 1. A origem floral dos méis avaliados deriva da combinação de espécies de plantas nativas, como Baccharis spp., Ilex paraguariensis A. St.-Hil., Mimosa scabrella Benth. e plantas exóticas cultivadas, como Eucalyptus spp. e Delonix regia (Bojer ex Hook.) Raf. (dados não apresentados).

\section{TABELA 1 - ESPECIFICAÇÕES DAS AMOSTRAS DE MEL DE MELIPONÍNEOS EM ESTUDO, DESTACANDO A ESPÉCIE PRODUTORA E O MUNICÍPIO DE PROCEDÊNCIA NO ESTADO DO PARANÁ (BRASIL)}

\begin{tabular}{|c|c|c|c|}
\hline Amostra & Espécie & Nome popular & Procedência \\
\hline $1-4$ & Melipona bicolor Lepeletier, 1836 & Guaraipo & Curitiba $(1,2)$; Guaraqueçaba $(3,4)$ \\
\hline $5-7$ & $\begin{array}{c}\text { Melipona marginata Lepeletier, } \\
1836\end{array}$ & Manduri & $\begin{array}{l}\text { Curitiba (5); Boa Esperança do Iguaçu } \\
\text { (6); Guaraqueçaba (7) }\end{array}$ \\
\hline $8-11$ & $\begin{array}{l}\text { Melipona quadrifasciata } \\
\text { Lepeletier, } 1836\end{array}$ & Mandaçaia & $\begin{array}{c}\text { Curitiba (8); Guaraqueçaba }(9,10) \text {; Boa } \\
\text { Esperança do Iguaçu }(11)\end{array}$ \\
\hline $12-13$ & $\begin{array}{l}\text { Melipona rufiventris } \\
\text { Lepeletier, } 1836\end{array}$ & Tujuba & Guaraqueçaba \\
\hline 14 & $\begin{array}{l}\text { Scaptotrigona ipunctata } \\
\text { Lepeletier, } 1836\end{array}$ & Tubuna & Mandirituba \\
\hline 15 & $\begin{array}{c}\text { Scaptotrigona xantotricha } \\
\text { Moure, } 1950\end{array}$ & Tujumirim & Guaraqueçaba \\
\hline $16-19$ & $\begin{array}{l}\text { Tetragona clavipes } \\
\text { Fabricius, } 1804\end{array}$ & Borá & $\begin{array}{c}\text { Boa Esperança do Iguaçu }(16,17) \text {; } \\
\text { Cornélio Procópio (18); Guraqueçaba } \\
\text { (19) }\end{array}$ \\
\hline $20-21$ & $\begin{array}{l}\text { Tetragonisca angustula } \\
\text { Latreille, } 1811\end{array}$ & Jataí & $\begin{array}{c}\text { Boa Esperança do Iguaçu (20); } \\
\text { Guaraqueçaba (21) }\end{array}$ \\
\hline
\end{tabular}




\subsubsection{Obtenção dos extratos}

Com o propósito de explorar o potencial antimicrobiano dos compostos fenólicos presentes nas amostras, extratos metanólicos foram elaborados pelo método de extração descrito por Ferreres et al. (1991), com modificações. Para cada amostra, $50 \mathrm{~g}$ de mel foram completamente misturados com $250 \mathrm{~mL}$ de água purificada, sendo o $\mathrm{pH}$ corrigido até $2,0 \mathrm{com} \mathrm{HCl}$ concentrado. Filtrou-se cada amostra para a remoção de partículas sólidas. O filtrado foi transferido para béquer contendo $50 \mathrm{~g}$ de resina Amberlite ${ }^{\circledR}$ XAD-2 (Fluka Chemie; poro: $9 \mathrm{~nm}$, partícula: 0,3-1,2 mm), permanecendo sob agitação por 15 minutos. O filtrado e a resina foram transferidos para uma coluna $(25 \times 2 \mathrm{~cm})$. Dessa forma, os compostos fenólicos ficaram retidos na resina, enquanto os açúcares e os outros compostos polares foram eluídos com solvente aquoso. Lavou-se a coluna com $100 \mathrm{~mL}$ de água acidificada e, em seguida, com $300 \mathrm{~mL}$ de água purificada. Toda a fração fenólica foi eluída com aproximadamente $300 \mathrm{~mL}$ de metanol até que desaparecesse a coloração da eluição e concentrada sob pressão reduzida à temperatura de $60^{\circ} \mathrm{C}$ para obtenção do extrato fenólico do mel (EFM). Os EFM foram, então, liofilizados.

\subsection{ATIVIDADE ANTIMICROBIANA}

\subsubsection{Micro-organismos e preparo do inóculo}

Todos os ensaios foram realizados em triplicata, utilizando-se cepas de Escherichia coli (ATCC 10530), Staphylococcus aureus (ATCC 25923) e Candida albicans (ATCC 10231), provenientes da American Type of Culture Collection (ATCC). Preparou-se o inóculo pela suspensão de colônias em solução salina até a turbidez da solução padrão de McFarland de 0,5 . A suspensão McFarland $0,5\left(1 \times 10^{8} \mathrm{UFC} \cdot \mathrm{mL}^{-1}\right)$ foi diluída $1: 10$ para se obter a diluição de $10^{7} \mathrm{UFC} . \mathrm{mL}^{-1}$. $\mathrm{O}$ volume de $0,005 \mathrm{~mL}$ dessa suspensão foi inoculado no caldo, sendo a concentração final de microorganismos do teste de aproximadamente $5 \times 10^{4}$ UFC.poço $^{-1}$.

\subsubsection{Determinação da concentração inibitória mínima (MIC) pelo método de microdiluição em caldo}

As amostras de mel foram diluídas a $50 \%$ no caldo Müeller-Hinton (CMH) para os testes com S. aureus e com E. coli e no caldo Müeller-Hinton com 2\% de glucose (CMHG) para os ensaios com C. albicans. Os EFM foram diluídos a $10 \mathrm{mg} \cdot \mathrm{mL}^{-1}$ nesses mesmos meios de culturas. Todas as soluções resultantes foram filtradas em membrana $0,22 \mu \mathrm{m}$, sendo os filtrados armazenados em tubos estéreis a $4^{\circ} \mathrm{C}$.

Determinou-se a ação da concentração inibitória mínima dos méis e dos EFM segundo o método de microdiluição em caldo, proposta pelo NCCLS (2003). Foram utilizadas microplacas com fundo em "U", esterilizadas, com 96 orifícios (TPP ${ }^{\circledR}$, EUA). Colocou-se o volume de $100 \mu \mathrm{L}$ de cada mel a $50 \%$ em um poço da micro-placa e, em seguida, $5 \mu \mathrm{L}$ de cada inóculo microbiano. A mesma quantidade de inóculo foi utilizada para as diluições seriadas de 25 a $0,024 \%$ dos méis de abelhas indígenas sem ferrão. Procedimento similar foi empregado para os extratos, sendo que ao volume de $100 \mu \mathrm{L}$ de cada EFM previamente adicionado em cada poço da microplaca nas diluições seriadas de 10 a $0,3125 \mathrm{mg} \cdot \mathrm{mL}^{-1}$, foram acrescidos $5 \mu \mathrm{L}$ de cada inóculo microbiano. Com o propósito de estabelecer comparação avaliou-se a atividade antimicrobiana de soluções de açúcares compostas por glucose e frutose em proporções iguais (BORSATO, CRUZ e ALMEIDA, 2009), nas concentrações de 70,65, 60, 55, 50 e 45\% (m/ ) , diluídas em CMH e CMHG para o volume final de $100 \mu \mathrm{L}$. Em cada uma dessas soluções também foram adicionados $5 \mu \mathrm{L}$ de inóculo microbiano.

As microplacas foram tampadas e incubadas a $35^{\circ} \mathrm{C}$ por $24 \mathrm{~h}$. A leitura ocorreu antes e após $24 \mathrm{~h}$ de incubação em leitor de microplacas (Biotek Instruments, Winooski, VT, EUA) a 
$405 \mathrm{~nm}$. Como controle positivo, avaliou-se o crescimento microbiano nos meios de cultura sem a adição de amostra. Considerando a possível influência da coloração das amostras na leitura espectrofotométrica, diluições dos méis, dos EFM e das soluções de açúcares em CMH e CMHG, na ausência de inóculo microbiano, foram avaliadas após $24 \mathrm{~h}$ de incubação. Os resultados dos ensaios antimicrobianos foram expressos como $\mathrm{MIC}_{90}$, concentração de amostra $(\mathrm{m} / \mathrm{V})$ capaz de inibir o crescimento microbiano em $90 \%$.

\section{RESULTADOS E DISCUSSÃO}

Estão sumarizados na Tabela 2 os resultados de $\mathrm{MIC}_{90}$, obtidos nos ensaios de atividade antimicrobiana (pelo método de microdiluição em caldo) para as amostras de mel produzidas pelas espécies de abelhas nativas sem ferrão M. bicolor, M. marginata, M. quadrifasciata, M. rufiventris, S. bipunctata, S. xantotricha, T. clavipes e T. angustula.

\section{TABELA 2 - VALORES DE MIC ${ }_{90}$ PARA OS MÉIS ELABORADOS PELAS ESPÉCIES DE ABELHAS INDÍGENAS SEM FERRÃO EM ESTUDO}

\begin{tabular}{cccc}
\hline \multirow{2}{*}{$\begin{array}{c}\text { Amostra de } \\
\text { mel }\end{array}$} & \multicolumn{3}{c}{ MIC $_{\mathbf{9}}(\% \mathbf{~ m} / \mathbf{V})$} \\
\cline { 2 - 4 } & Escherichia coli & Staphylococcus aureus & Candida albicans \\
\hline 1 & 25 & 25 & 50 \\
2 & 25 & 25 & 50 \\
3 & 12,5 & 12,5 & 25 \\
4 & 12,5 & 12,5 & 25 \\
5 & 25 & 12,5 & 50 \\
6 & 25 & 25 & 50 \\
7 & 12,5 & 12,5 & 50 \\
8 & 6,25 & 3,13 & 25 \\
9 & 25 & 50 & 50 \\
10 & 12,5 & 12,5 & 25 \\
11 & 25 & 25 & 50 \\
12 & 25 & 25 & 50 \\
13 & 25 & 50 & 50 \\
14 & 3,12 & 1,56 & 12,5 \\
15 & 25 & 25 & 50 \\
16 & 25 & 25 & 50 \\
17 & 12,5 & 12,5 & 25 \\
18 & 12,5 & 25 & 25 \\
19 & 12,5 & 25 & 25 \\
20 & 6,25 & 6,25 & 25 \\
21 & 50 & 50 & 50 \\
\hline
\end{tabular}

É possível observar que os três micro-organismos avaliados foram sensíveis as 21 amostras de mel de meliponíneos nas concentrações testadas, sendo que, em geral, as bactérias E. coli e S. aureus mostraram-se mais sensíveis. Para as amostras codificadas de 1 a 20 foram verificados valores de $\mathrm{MIC}_{90}$ mais elevados para a levedura C. albicans do que para E. coli e S. aureus. Tal fato indica que esse micro-organismo é comparativamente mais resistente à ação dos compostos antimicrobianos presentes nas amostras de mel em avaliação, como previamente já relatado na literatura (DARDÓN e ENRÍQUEZ, 2008; BOORN et al., 2010). Apenas a amostra 21, proveniente de $T$. angustula, apresentou os mesmos valores de $\mathrm{MIC}_{90}$ para os três micro-organismos. 
Os resultados da atividade antimicrobiana evidenciados para os extratos fenólicos elaborados a partir dos méis de abelhas indígenas sem ferrão estão apresentados na Tabela 3.

\section{TABELA 3 - VALORES DE MIC ${ }_{90}$ PARA OS EXTRATOS FENÓLICOS DOS MÉIS PRODUZIDOS PELAS ESPÉCIES DE ABELHAS INDÍGENAS SEM FERRÃO EM ESTUDO}

\begin{tabular}{|c|c|c|c|}
\hline \multirow{2}{*}{ EFM } & \multicolumn{3}{|c|}{$\mathrm{MIC}_{90}\left(\mathrm{mg} \cdot \mathrm{mL}^{-1}\right)$} \\
\hline & Escherichia coli & Staphylococcus aureus & Candida albicans \\
\hline 1 & $>10$ & $>10$ & $>10$ \\
\hline 2 & $>10$ & 10 & $>10$ \\
\hline 3 & $>10$ & $>10$ & $>10$ \\
\hline 4 & $>10$ & 10 & $>10$ \\
\hline 5 & $>10$ & $>10$ & $>10$ \\
\hline 6 & $>10$ & 10 & $>10$ \\
\hline 7 & $>10$ & $>10$ & $>10$ \\
\hline 8 & $>10$ & 10 & $>10$ \\
\hline 9 & $>10$ & $>10$ & $>10$ \\
\hline 10 & $>10$ & $>10$ & $>10$ \\
\hline 11 & $>10$ & $>10$ & $>10$ \\
\hline 12 & $>10$ & $>10$ & $>10$ \\
\hline 13 & $>10$ & $>10$ & $>10$ \\
\hline 14 & $>10$ & 10 & $>10$ \\
\hline 15 & $>10$ & 10 & $>10$ \\
\hline 16 & $>10$ & 10 & $>10$ \\
\hline 17 & $>10$ & $>10$ & $>10$ \\
\hline 18 & $>10$ & $>10$ & $>10$ \\
\hline 19 & $>10$ & 10 & $>10$ \\
\hline 20 & $>10$ & $>10$ & $>10$ \\
\hline 21 & $>10$ & 10 & $>10$ \\
\hline
\end{tabular}

Nas condições avaliadas, os EFM revelaram menor potencial antimicrobiano que os méis in natura. Para nove amostras de EFM, apenas o crescimento de $S$. aureus foi inibido em $90 \%$ quando se utilizou $10 \mathrm{mg} \cdot \mathrm{mL}^{-1}$. Os micro-organismos $E$. coli e $C$. albicans demonstraram-se resistentes à ação dos EFM nas concentrações testadas.

Com a finalidade de verificar se o efeito osmótico, isoladamente, seria capaz de inibir o crescimento de micro-organismos, também foi investigada a atividade antimicrobiana de solução de açúcares contendo iguais proporções de glucose e frutose. Observou-se inibição do crescimento em $90 \%$ apenas para E. coli em soluções de açúcares em concentração maior ou igual a $50 \%$. Para os micro-organismos S. aureus e C. albicans, entretanto, não houve inibição do crescimento nas concentrações testadas. Isso demonstra que a alta pressão osmótica pode exercer efeito inibidor do crescimento de E. coli. Porém, o potencial antimicrobiano apresentado pelos méis investigados não pode ser atribuído somente ao efeito osmótico contra $E$. coli, pois as amostras foram diluídas inicialmente a $50 \%(\mathrm{~m} / \mathrm{V})$. Nessa diluição, o mel tem a sua concentração 
de açúcares reduzida à metade. Considerando que o mel produzido por algumas espécies do gênero Melipona apresenta teor médio de açúcares de, aproximadamente, $71 \%$ em sua composição (SOUZA et al., 2004b), a concentração de açúcares na diluição inicial não ultrapassou $40 \%$. Assim, tal concentração não seria suficiente para fornecer os resultados obtidos para esse micro-organismo.

Os méis de M. quadrifasciata (amostra 8), S. bipunctata (amostra 14) e T. angustula (amostra 20) inibiram o crescimento de E. coli e S. aureus em menores concentrações, sendo, portanto, mais interessantes como alimentos com propriedades antibacterianas. Além disso, o mel de S. bipunctata apresentou a menor $\mathrm{MIC}_{90}$ para os três micro-organismos avaliados, destacandose como o melhor agente antibacteriano e antifúngico comparativamente aos demais méis investigados.

A partir dos resultados obtidos pode-se inferir que a ação antimicrobiana não decorre somente da espécie de abelha sem ferrão produtora do mel, uma vez que diferentes amostras elaboradas pelo mesmo meliponíneo demonstraram comportamento diferenciado em relação ao efeito analisado. Nesse sentido, a composição química particular de cada amostra, bem como os diferentes aspectos que a influenciam, podem ter desempenhado papel decisivo na atividade biológica. Conforme a literatura (WESTON, 2000), a variação na ação antimicrobiana se deve a muitos fatores que afetam a composição química dos méis, incluindo diferenças no solo e nas condições atmosféricas, além das diversas espécies vegetais que contribuem para a formação desses produtos.

Vários fatores podem concorrer para a propriedade antimicrobiana do mel, dentre os quais se encontram: baixa atividade de água $\left(\mathrm{A}_{\mathrm{w}}\right)$, alta pressão osmótica, baixo $\mathrm{pH}$, o sistema glucose/ oxidase com formação de peróxido de hidrogênio, alta taxa carbono/nitrogênio, baixo potencial redox relacionado ao alto conteúdo de açúcares redutores, baixo conteúdo de proteínas e a presença de componentes fitoquímicos e de substâncias voláteis (HOOPER, 1976; WHITE, 1979; MOLAN, 1997; WESTON, 2000; COOPER, MOLAN e HARDING, 2002). Essas diferentes qualidades, em sinergismo, podem ter sido responsáveis pelos efeitos antibacterianos e antifúngicos verificados para os méis avaliados.

O mel produzido pelas abelhas sem ferrão difere em muitos aspectos do mel elaborado por abelhas do gênero Apis L. Os meliponíneos apresentam característica particular relevante, pois armazenam o mel em potes de cerume que são feitos de cera combinada com própolis (BALLIVIÁN, 2008), enquanto que a abelha Apis mellifera L. armazena o seu mel em favos elaborados somente com cera (PERSANO-ODDO et al. 2008). Diante do exposto e considerando que o mel de abelha sem ferrão, durante o seu armazenamento, permanece em contato com a própolis, rica em resinas de plantas, há grande possibilidade de que esses constituintes fitoquímicos sejam infundidos ao mel, conferindo-Ihe propriedades antibacterianas, antifúngicas e antivirais (TEMARU et al., 2007). As espécies botânicas que contribuem para a composição da própolis também determinam a composição química do mel, o que pode justificar as diferenças na ação antimicrobiana encontradas para amostras provenientes da mesma espécie de abelha sem ferrão.

Diferentemente do verificado para as amostras de mel de meliponíneos in natura, os EFM indicaram menor efeito antimicrobiano. Weston, Brocklebank e Lu (2000) investigaram o perfil cromatográfico da fração fenólica e a atividade antibacteriana de 19 amostras de mel de manuka (Leptospermum scoparium Forst.) e demonstraram que os compostos fenólicos, isoladamente, não são os principais componentes responsáveis pela presença/ausência da atividade antibacteriana. Os resultados obtidos no presente trabalho estão de acordo com a literatura, pois somente um terço dos EFM extraídos das amostras de mel de abelhas sem ferrão dos gêneros Melipona, Scaptotrigona, Tetragona e Tetragonisca, apresentou ação antibacteriana e apenas para S. aureus. Assim, é possível postular que os compostos fenólicos representam contribuição minoritária e parcial ao efeito antimicrobiano dos méis de meliponíneos. 
A partir dos resultados obtidos no presente estudo é possível propor a indicação dos méis avaliados de Melipona, Scaptotrigona, Tetragona e Tetragonisca como alimentos com propriedades funcionais, com aplicação no tratamento de infecções causadas por E. coli, S. aureus e C. albicans. Esses resultados também apoiam a alegação medicinal para o mel de abelhas nativas sem ferrão, como aquela já atribuída ao mel de $A$. melifera.

\section{CONCLUSÃO}

As amostras de mel de abelhas indígenas sem ferrão de Melipona, Scaptotrigona, Tetragona e Tetragonisca, provenientes do Estado do Paraná (Brasil), apresentaram atividade antimicrobiana para cepas de E. coli, S. aureus e C. albicans. De forma comparativa, a amostra de mel de $S$. bipunctata destacou-se como o melhor agente antimicrobiano. Os EFM indicaram reduzido efeito antimicrobiano em relação aos méis de meliponíneos.

Os resultados obtidos constituem base experimental para no futuro, e mediante estudos adicionais, propor o uso desse alimento in natura como produto natural promissor para o tratamento de processos infecciosos causados por bactérias e fungos.

\section{ABSTRACT \\ ANTIMICROBIAL ACTIVITY OF HONEYS PROVIDED BY MELIPONINES FROM PARANÁ (BRAZIL)}

The aim of this paper was to study the antibacterial and antifungal activity of pure honeys provided by stingless bees and phenolic extracts (HPES) obtained from these samples. A total of 21 honey samples were collected in Paraná (Brazil). The antimicrobial activity was assessed by broth microdilution. Minimum inhibitory concentration (MIC) was determined against Escherichia coli ATCC10530, Staphylococcus aureus ATCC 25923 and Candida albicans ATCC 10231. The honey samples presented higher antimicrobial activity against E. coli (MIC90 $\geq 3.12 \%$ ) and S. aureus (MIC90 $\geq 1.56 \%$ ) as compared to C. albicans (MIC90 $\geq 12.5 \%$ ). A lower antimicrobial activity was verified for HPES in relation to pure honeys. Only nine samples of HPE inhibited the growth of $S$. aureus at $10 \mathrm{mg} \cdot \mathrm{mL}^{-1}$. Thus it is possible to suggest that stingless bee honey is a food with a health claim and has therapeutic potential against infections.

KEY-WORDS: STINGLESS BEE; HONEY; PHENOLIC EXTRACT; INFECTION; BROTH MICRODILUTION.

\section{REFERÊNCIAS}

1 ALMEIDA-MURADIAN, L.B. de; MATSUDA, A.H.; BASTOS, D.H.M. Physicochemical parameters of amazon melipona honey. Química Nova, v. 30, n. 3, p. 707-708, 2007.

2 ALVAREZ-SUAREZ, J.M.; TULIPANI, S.; ROMANDINI, S.; BERTOLI, E.; BATTINO, M. Contribution of honey in nutrition and human health: a review. Mediterranean Journal of Nutrition and Metabolism, v. 3, p. 15-23, 2010.

3 ALVES, D.F.S.; CABRAL JÚNIOR, F. das C.; CABRAL, P.P. de A.C.; OLIVEIRA JÚNIOR, R.M. de; REGO, A.C.M. do; MEDEIROS, A.C. Efeitos da aplicação tópica do mel de Melipona subnitida em feridas infectadas de ratos. Revista do Colégio Brasileiro de Cirurgiões, v. 35, p. 188-193, 2008.

4 BALLIVIÁN, J.M.P.P. Abelhas nativas sem ferrão. São Leopoldo: Oikos, 2008. 128 p.

5 BOGDANOV, S.; JURENDIC, T.; SIEBER, R.; GALLMAN, P. Honey for nutrition and health: a review. American Journal of the College of Nutrition. v. 27, p. 677-689, 2008.

6 BOORN, K.L.; KHOR, Y.Y.; SWEETMAN, E.; TAN, F.; HEARD, T.A.; HAMMER, K.A. Antimicrobial activity of honey from the stingless bee Trigona carbonaria determined by agar diffusion, agar dilution, broth microdilution and time-kill methodology. Journal of Applied Microbiology, v. 108, p. 1534-1543, 2010.

7 BORSATO, D.M.; CRUZ, M.C.R. da; ALMEIDA, M. de. Atividade antimicrobiana de méis comercializados na região dos Campos Gerais - Paraná. Visão Acadêmica, v.10, p. 48-53, 2009.

8 BORSATO, D.M.; VARGAS, T.; KOOP, L.; FARAGO, P.V.; ALMEIDA, M. de. Physicochemical quality control of bee honeys from Campos Gerais region of Paraná - Brazil. Boletim do CEPPA, v. 28, n. 2, p. 205-212, 2010. 
9 COOPER, R.A.; MOLAN, P.C.; HARDING, K.G. The sensitivity to honey of Gram-positive cocci of clinicance isolated from wounds. Journal of Applied Microbiology, v.93, p. 857-863, 2002.

10 CORTOPASSI-LAURINO, M.; GELLI, D.S. Analyse pollinique, propriétés physico-chimiques et action des mieis d'abellies africanisées Apis mellifera et de Méliponinés du Brésil. Apidologie, v.22, p.61-73, 1991.

11 CRANE, E. The past and present importance of bee products to men. In: MIZRAHI, A.; LENSKY, Y. (Ed). Bee products: properties, applications and apitherapy. New York: Plenum Press, 1997. p. 1-14.

12 DARDÓN, M.J; ENRÍQUEZ, E. Caracterización fisicoquímica y antimicrobiana de la miel de nueve especies de abejas sin aguijón (Meliponini) de Guatemala. Interciencia, v. 33, p. 916-922, 2008.

13 FERRERES, F.; TOMÁS-BARBERÁN, F.A.; GIL, M.I.; TOMÁS-LORENTE, F. An HPLC technique for flavonoid analisys in honey. Journal of the Science of Food and Agriculture, v. 56, p. 49-56, 1991.

14 GAREDEW, A.; SCHMOLZ, E.; LAMPRECHT, I. Microcalorimetric investigation on the antimicrobial activity of honey of the stingless bee Trigona spp. and comparison of some parameters with those obtained with standard methods. Thermochimica Acta, v. 415, p. 99-106, 2004

15 GONÇALVES, A.L.; ALVES FILHO, A.; MENEZES, H. Atividade antimicrobiana do mel da abelha nativa sem ferrão Nannotrigona testaceicornis (Hymenoptera: Apidae, Meliponini). Arquivos do Instituto Biológico, v. 72, p. 455-459, 2005.

16 GUERRINI, A.; BRUNI, R.; MAIETTI, S.; POLI, C.; ROSSI, D.; PAGANETTO, G.; MUZZOLI, M.; SCALVENZI, L.; SACCHETTI, G. Ecuadorian stingless bee (Meliponinae) honey: a chemical and functional profile of an ancient health product. Food Chemistry, v. 114, p. 1413-1420, 2009.

17 HOOPER, T. Guia do apicultor. [S.I.]: Publicações Europa-América, 1976. p. 223-266.

18 HSUEH, P.; CHEN, W.; LUH, K. Relationships between antimicrobial use and antimicrobial resistance in Gram-negative bacteria causing nosocomial infections from 1991-2003 at a university hospital in Taiwan. International Journal of Antimicrobial Agents, v. 26, p. 463-472, 2005.

19 IRISH, J.; CARTER, D.A.; BLAIR, S.E.; HEARD, T.A. Antibacterial activity of honey from the Australian stingless bee Trigona carbonaria. International Journal of Antimicrobial Agents, v. 32, p. 89-90, 2008.

20 IRISH, J.; CARTER, D.A.; SHOKOHI, T.; BLAIR, E.S. Honey has an antifungal effect against Candida species. Medical Mycology, v. 44, p. 289-291, 2006.

21 MARCHINI, L.C.; MORETI, A.C. de C.C.; SILVEIRA NETO, S. Características físico-químicas de amostras de mel e desenvolvimento de enxames de Apis mellifera L., 1758 (Hymenoptera, Apidae) em cinco diferentes espécies de eucaliptos. Boletim do CEPPA, v. 21, n. 1, p. 193-206, 2003.

22 MIORIN, P.L.; LEVY JUNIOR, N.C.; CUSTODIO, A.R.; BRETZ, W.A.; MARCUCCI, M.C. Antibacterial activity of honey and propolis from Apis mellifera and Tetragonisca angustula against Staphylococcus aureus. Journal of Applied Microbiology, v. 95, p. 913-920, 2003.

23 MOLAN, P.C. Honey as an antimicrobial agent. In: MIZRAHI, A; LENSKY, Y. (Eds). Bee products: properties, applications and apitherapy. New York: Plenum Press, 1997. p. 27-37.

24 MOLAN, P.C.; SMITH, I.M.; REID, G.M. A comparison of the antibacterial activity of some New Zealand honeys. Journal of Apicultural Research, v. 27, p. 252-256, 1988.

25 NATIONAL COMMITTEE FOR CLINICAL LABORATORY STANDARDS (NCCLS). Methods for dilution antimicrobial susceptibility tests for bacteria that grow aerobically. $6^{\text {th }}$ ed. Wayne, Pennsylvania, 2003. (NCCLS document M7-A6).

26 PERSANO-ODDO, L.; HEARD, T. A.; RODRÍGUES-MALAVER, A.; PÉREZ, R. A.; FERNÁNDEZ-MUIÑO, M.; SANCHO, M. T.; SESTA, G.; LUSCO, L.; VIT, P. Composition and antioxidant activity of Trigonia carbonaria honey from Australia. Journal of Medicinal Food, v. 11, p. 789-794, 2008.

27 POSEY, D.A. Etnoentomologia de tribos indígenas da Amazônia. In: RIBEIRO, D. (Ed.) Suma etnológica brasileira. 2.ed Petrópolis: FINEP/Vozes, 1987. p. 251-271.

28 SOUZA, B.; CARVALHO, C.A.L.; SODRÉ, G.S.; MARCHINI, L.C. Características físico-químicas de amostras de mel de Melipona asilvai (Hymenooptera: Apidae). Ciência Rural, v. 34, p.1623-1624, $2004 a$.

29 SOUZA, R.C. da S.; YUYAMA, L.K.O.; AGUIAR, J.P.L.; OLIVEIRA, F.P.M. Valor nutricional do mel e pólen de abelhas sem ferrão da região amazônica. Acta Amazonica, v. 34, p. 333-336, 2004 b.

30 TEMARU, E.; SHIMURA, S.; AMANO, K.; KARASAWA, T. Antibacterial activity of honey from stingless honeybees (Hymenoptera: Apidae; Meliponinae). Polish Journal of Microbiology, v. 56, p. 281-285, 2007. 
31 TORRES, A.; GARADEW, A.; SCHMOLZ, E.; LAMPRECHT, I. Calorimetric investigation of the antimicrobial action and insight into the chemical properties of "angelita" honey - a product of the stingless bee Tetragonisca angustula from Colombia. Thermochimica Acta, v. 415, p. 107-113, 2004.

32 WESTON, R.J.; BROCKLEBANK, L.K.; LU, Y. Identification and quantitative levels of antibacterial components of some New Zealand honeys. Food Chemistry, v. 70, p. 427-435, 2000.

33 WESTON, R.J. The contribution of catalase and others natural products to the antibacterial activity of honey: a review. Food Chemistry, v. 71, p. 235-239, 2000.

34 WHITE, J.W. Composition of honey. In: CRANE, E. Honey: a comprehensive survey. Londres: Heinemann, 1979. p. 157207.

\section{AGRADECIMENTO}

À CAPES-REUNI pela concessão da bolsa de estudos. 